\title{
Prevention of the development of adverse reactions in the treatment of tuberculosis in combination with diabetes mellitus
}

\section{Yeremenchuk I.V.}

Bukovinian State Medical University, Chernivtsi, Ukraine

Background. In recent decades, tuberculosis (TB) and its resistant forms have become an increasing problem in lowincome countries, especially in countries with epidemics of HIV infection and insulin-dependent diabetes mellitus (DM). DM has been shown to adversely affect TB treatment outcomes. Low treatment outcomes for TB/DM comorbidity are associated with age, weight gain, or poor glycemic control, and it is the increased duration of DM treatment that may be the best strategy for improving outcomes in TB/DM patients.

In the formation of the development of polyneuropathy, one of the components is increasing ischemia and intracellular hypoxia, which is characteristic of TB/DM. Decreased blood flow contributes to the destabilization of cell membranes and neuronal death. The interconnected components of the immune and other systems, namely pro-inflammatory cytokines, play an important role in the formation and progression of these processes.

Results and discussion. Our clinical assessment of the general condition of patients with TB/DM (23 patients) showed that $91.5 \%$ of the examined patients complained of persistent cough with sputum production, $78 \%$ had shortness of breath, weight loss of more than $5 \mathrm{~kg}-89.8 \%$, thirst - $80 \%, 75 \%$ tingling and numbness of the lower extremities, pain in the legs at night $-85.5 \%$ of people. Sub-febrile temperature (37.4-37.9 ${ }^{\circ} \mathrm{C}$ ) was registered in $52.2 \%$ of the examined patients. The general condition of moderate severity prevailed in $49.5 \%$ of patients. Intoxication syndrome (IS) in TB/DM patients by clinical and paraclinical signs was observed in all patients, respectively, pronounced (manifest) IS was recorded in $79.5 \%$. Body mass index is on average $15.6 \mathrm{~kg} / \mathrm{m}^{2}(p<0.001)$.

To prevent the development of polyneuropathy, patients with TB/DM was prescribed anti-TB drugs, insulin and additionally pathogenetic therapy according to the scheme: Neuromax, $2 \mathrm{ml}$ intravenously, intramuscular once a day for 7 days, followed by the administration of the drug 2 times a week for 3 weeks, and with the subsequent transition to the tablet form - 1 tablet per day (until the end of the intensive phase of treatment), then Dialipon $3 \%$ solution of $20 \mathrm{ml}$ was prescribed intravenously drip for 7 days (the contents of the ampoule are diluted in $250 \mathrm{ml}$ of $0.9 \%$ sodium chloride solution), with the subsequent transition to a tablet form of $300 \mathrm{mg}-2$ tablets once a day for a month.

Conclusions. The proposed method makes it possible to effectively prevent adverse reactions in the treatment of TB in combination with DM, reduce the manifestations of peripheral polyneuropathy, provide better tolerance to anti-TB drugs, normalize clinical blood parameters, accelerate the rate of normalization of the content of some pro (IL-6, IL-18) and anti-inflammatory (IL-10) cytokines in the blood plasma of patients, which, in turn, reduces the percentage of refusals from the treatment of comorbid pathology. 